Revista Baiana de Saúde Pública

ARTIGO ORIGINAL DE TEMA LIVRE

\title{
ANÁLISE DA IMPLANTAÇÃO DO SISTEMA DE CADASTRAMENTO E ACOMPANHAMENTO DE HIPERTENSOS E DIABÉTICOS EM MUNICÍPIOS DA BAHIA - 2013
}

Edna Pereira Rezende ${ }^{a}$ Luis Eugênio Portela Fernandes de Souza ${ }^{b}$

\begin{abstract}
Resumo
O Sistema Único de Saúde conta com o programa HiperDia, específico para enfrentar o problema da hipertensão e do diabetes, o qual contempla um sistema de informações próprio, o SIS-HiperDia. O objetivo deste trabalho foi analisar o processo de implantação do SIS-HiperDia em municípios selecionados no estado da Bahia. Trata-se de estudo de avaliação, em que foi desenvolvido um dos seus componentes, a estimativa do grau de implantação, com a análise dos seus determinantes contextuais. Coordenadores da atenção básica de 117 municípios responderam acerca das condições de implantação do sistema. As análises foram realizadas com base nos modelos lógico e de contexto elaborados previamente. Os resultados apontaram que o SIS-HiperDia foi implantado em apenas 43,6\% dos municípios analisados. A implantação foi influenciada por elementos contextuais ligados a questões gerenciais e operacionais. As fragilidades identificadas, sobretudo, podem ajudar os gestores a desenvolverem estratégias para melhorar a qualidade da informação e usá-las como ferramenta de planejamento e de gestão do cuidado em saúde. Concluiu-se que o grau de implantação do SIS-HiperDia nos municípios baianos analisados foi baixo.
\end{abstract}

Palavras-chave: Hipertensão arterial. Diabetes mellitus. Análise de implantação de programas. SIS-HiperDia.

Secretaria de Saúde do Estado da Bahia. Salvador, Bahia, Brasil.

b Universidade Federal da Bahia/Instituto de Saúde Coletiva. Salvador, Bahia, Brasil. Endereço para correspondência: Conj Resid DNER BR 324, km 620, casa 3, Campinas/Pirajá, Salvador, Bahia, Brasil. CEP: 41275-120. E-mail: dantdiveprezende@gmail.com 


\title{
ANALYSIS OF THE IMPLEMENTATION OF THE REGISTRATION AND MONITORING OF \\ HYPERTENSIVE AND DIABETICS SYSTEM IN MUNICIPALITIES OF BAHIA - 2013
}

\begin{abstract}
The Unified Health System has the HiperDia program, specific to address the problem of hypertension and diabetes, which includes a proper information system, SISHiperDia. The objective of this work was to analyze the SIS-HiperDia implementation process in selected municipalities in the state of Bahia. It is an evaluation study, in which one of its components was developed, the estimation of the degree of implementation, with the analysis of its contextual determinants. Coordinators of the basic attention of 117 municipalities answered about the conditions of implantation of the system. The analysis were carried out based on logical and context models previously elaborated. The results showed that SIS-Hiperdia was implanted in only $43.6 \%$ of the municipalities analyzed. The implementation was influenced by contextual elements related to managerial and operational issues. The identified fragilities, above all, can help managers developing strategies to improve the quality of information and use them as a health care planning and management tool. The conclusion is that the degree of SIS-HiperDia implantation in the analyzed municipalities of Bahia was low.
\end{abstract}

Keywords: Hypertension. Diabetes Mellitus. Analysis of the implementation of programs. SIS-HiperDia.

\section{ANÁLISIS DE LA IMPLEMENTACIÓN DEL SISTEMA DE REGISTRO Y SEGUIMIENTO DE HIPERTENSOS Y DIABETICOS EN MUNICIPIOS DE BAHIA - 2013}

\section{Resumen}

El sistema único de salud tiene el programa HiperDia, específico para enfrentar los problemas de la hipertensión y de la diabetes, que comprende un sistema de información propio, SIS-HIPERDIA. El objetivo de este estudio fue analizar el proceso de implementación de SIS-HIPERDIA en determinados municipios en el estado de Bahia. Este es un estudio de evaluación, en que un de sus componentes fue desarrollado, la estimación del grado de implantación, con el análisis contextual de sus determinantes. Los coordinadores de la atención primaria de alrededor de 117 municipios respondieron sobre las condiciones de implementación del sistema. Realizaronse análisis basados en los modelos lógicos y de contexto previamente elaborados. Los resultados mostraron que se implantó SIS-HIPERDIA 
Revista Baiana de Saúde Pública en sólo el 43,6\% de los municipios analizados. La implantación fue influenciada por factores contextuales relacionados con cuestiones gerenciales y operacionales. Las debilidades identificadas, sobre todo, pueden ayudar los gerentes a desarrollar estrategias para mejorar la calidad de la información y utilizarla como una herramienta de planificación y administración de la salud. Concluyese que el nivel de implantación de SIS-HIPERDIA en los municipios analizados en Bahia fue bajo.

Palabras clave: Hipertensión arterial. La diabetes mellitus. Análisis de implementación de programas. SIS-HiperDia.

\section{INTRODUÇÃO}

As doenças crônicas não transmissíveis (DCNT) são um problema de saúde global e respondem por elevada morbimortalidade em muitos países, sejam eles de alta, média ou baixa condição socioeconômica ${ }^{1}$. Representam um alto custo para os sistemas de saúde de todo o mundo, com grande impacto econômico para os portadores, suas famílias e também a sociedade². Estimativa da Organização Mundial de Saúde (OMS) aponta que as DCNT são responsáveis por $58,5 \%$ de todas as mortes ocorridas no mundo e por $45,9 \%$ da carga global de doenças ${ }^{3-4}$.

A hipertensão arterial sistêmica (HAS) e o diabetes mellitus (DM) estão entre as DCNT mais comuns ${ }^{1}$. Constituem os principais fatores de risco para as doenças do aparelho circulatório, apresentam processo de instalação insidioso e assintomático, têm histórias naturais prolongadas, multiplicidade de fatores de risco, evolução para graus variados de incapacidade ou para morte e acometem principalmente a população adulta. A hipertensão arterial é um problema crônico bastante frequente, com prevalências que variam de 19,2\% a 44,4\% ${ }^{5}$. No Brasil, segundo dados do Inquérito Telefônico de Vigilância de Fatores de Risco e Proteção para Doenças Crônicas, 24,1\% dos indivíduos a partir dos 18 anos referiram ser hipertensos ${ }^{6}$. O DM configura-se hoje como epidemia mundial e suas consequências humanas, sociais e econômicas são devastadoras para o mundo. Do total de mortes por ano, 4 milhões (9\%) são causadas por essa doença e suas complicações ${ }^{7}$.

Também no Brasil, as DCNT tornaram-se grande problema de saúde pública. A elas são atribuídas $72 \%$ das mortes ocorridas em $2007^{2}$. Na Bahia, embora o número de óbitos prematuros (30 a 69 anos) pelas quatro principais doenças crônicas (doenças cardiovasculares, diabetes mellitus, doenças respiratórias crônicas e neoplasias) tenha aumentado entre 2007 e 2010, a taxa de mortalidade apresentou tendência decrescente no mesmo período ${ }^{8}$. 
O Brasil tem posto em prática importantes políticas de prevenção das DCNT e ampliado o escopo da ação do cuidado médico para a prevenção da doença, a promoção da saúde e a ação intersetorial. Entre as estratégias adotadas para reduzir o ônus das DCNT para a população brasileira, destacam-se as medidas antitabágicas, a exemplo de: criação de leis para controlar o tabaco e seu uso, além da proibição da propaganda de seus produtos; políticas de alimentação e nutrição e de promoção da saúde, com ênfase na escola; resolução que regulamenta a propaganda de alimentos ricos em açúcar, sal ou gorduras não saudáveis. Tomaram-se medidas importantes para o controle do consumo excessivo de álcool, como a regulamentação de propaganda e venda; cumprimento da lei para quem ingerir álcool e dirigir; e atendimento a indivíduos com problemas relacionados ao álcool, o que culminou na Política Nacional do Álcool, em $2007^{2}$.

É importante registrar a adoção da Estratégia de Saúde da Família como política prioritária de atenção básica que, por sua conformação e processo de trabalho, compreende as condições mais favoráveis de acesso às medidas multissetoriais e integrais que a abordagem DCNT exige. O trabalho das equipes de saúde da família pode contribuir para o cuidado integral e contínuo e, assim, propiciar uma plataforma para a prevenção e o gerenciamento das doenças crônicas 9 .

A Secretaria de Vigilância em Saúde (SVS/MS) desenvolve a vigilância de fatores de risco ou proteção para doenças crônicas por inquérito telefônico (Vigitel) desde 2006, com o objetivo de fazer o monitoramento contínuo da frequência e distribuição de fatores de risco ou proteção para as doenças crônicas em todas as capitais dos 26 estados e no distrito federal. Segundo dados do Vigitel 2012, a frequência de adultos que referiram diagnóstico médico de HAS variou de 16,6\% em Boa Vista a 29,7\% no Rio de Janeiro; para o DM, a frequência variou de 4,3\% em Palmas a 9,3\% em São Paulo. Em Salvador, as frequências foram 25,7\% para HAS e $6 \%$ para o $\mathrm{DM}^{10}$.

Também foram implementadas ações de atenção à hipertensão e ao diabetes notadamente na rede básica, com destaque para o Plano Nacional de Reorganização da Atenção à Hipertensão e ao Diabetes Mellitus (PNHD), a disponibilização dos medicamentos genéricos de baixo custo, como aspirina e estatinas, para indivíduos com alto risco atribuível de doenças cardíacas, a expansão pelo MS do programa Farmácia Popular, no início de 2011, o qual passou a oferecer gratuitamente medicamentos básicos para diabetes e hipertensão, além da implementação de diretrizes e materiais para o gerenciamento preventivo de outras DCNT².

O PNHD lançado pelo MS em 2001, entre os seus instrumentos, contemplava o Sistema HiperDia (SIS-HiperDia). Esperava-se que esse sistema informatizado de cadastro e 
Revista Baiana de Saúde Pública acompanhamento de hipertensos e diabéticos na rede básica, em médio prazo, pudesse gerar informações sobre o perfil epidemiológico dessa população e consequentemente subsidiar o desencadeamento de estratégias de saúde pública que levariam à modificação do cenário atual, à melhoria da qualidade de vida dessas pessoas e à redução dos custos assistenciais e sociais associados a esses agravos ${ }^{11}$.

Os dados disponíveis na base do SIS-HiperDia/DataSUS/MS em dezembro de 2013 sugeriam que esse sistema não alcançou, na Bahia, a meta de ser implantado e alimentado sistematicamente em todos os municípios. Assim, é grande o número de hipertensos e diabéticos não cadastrados, provavelmente não diagnosticados e não vinculados às unidades básicas de saúde e, portanto, sem o acompanhamento necessário ao controle dessas patologias e suas complicações. Na relação entre os dados do SIS-HiperDia e os do Sistema de Informação da Atenção Básica (SIAB), no qual a hipertensão e o diabetes são autorreferidos pelas famílias, observou-se que o número de hipertensos e diabéticos cadastrados nesse sistema estadual é significativamente superior ao do SIS-HiperDia, o que demonstra inconsistências entre esses sistemas. Considerando esse fato e que a análise dessa relação não agrega informações para estimar o grau de implantação, optou-se por não se utilizar os dados do SIAB neste estudo.

Não se sabe, contudo, em termos absolutos e relativos, o número exato de municípios que implantaram o SIS-HiperDia e, mais que isso, quais fatores ou aspectos contextuais influenciaram os diferentes graus de implantação alcançados pelos diversos municípios. Nesse sentido, a pesquisa buscou contribuir para a produção de conhecimento nessa área e subsidiar a implementação das políticas públicas voltadas à assistência aos hipertensos e diabéticos.

O objetivo deste trabalho foi analisar o processo de implantação do SIS-HiperDia em municípios selecionados no estado da Bahia.

\section{METODOLOGIA}

Trata-se de estudo de avaliação, em que foi desenvolvido um dos seus componentes: a estimativa do grau de implantação com a análise dos seus determinantes contextuais.

O primeiro passo para a produção dos dados foi a elaboração do modelo lógico (Figura 1), que contemplou os componentes, a estrutura, as atividades e os resultados intermediários e final do SIS-HiperDia; e o modelo de análise do contexto (Figura 2), que correlacionou o modelo lógico com determinantes que podem dificultar ou contribuir para o alcance dos resultados. A construção de ambos teve como fontes documentos oficiais ${ }^{11-13}$. 
Figura 1 - Modelo lógico do sistema de cadastro e acompanhamento de hipertensos e diabéticos / SIS-HiperDia

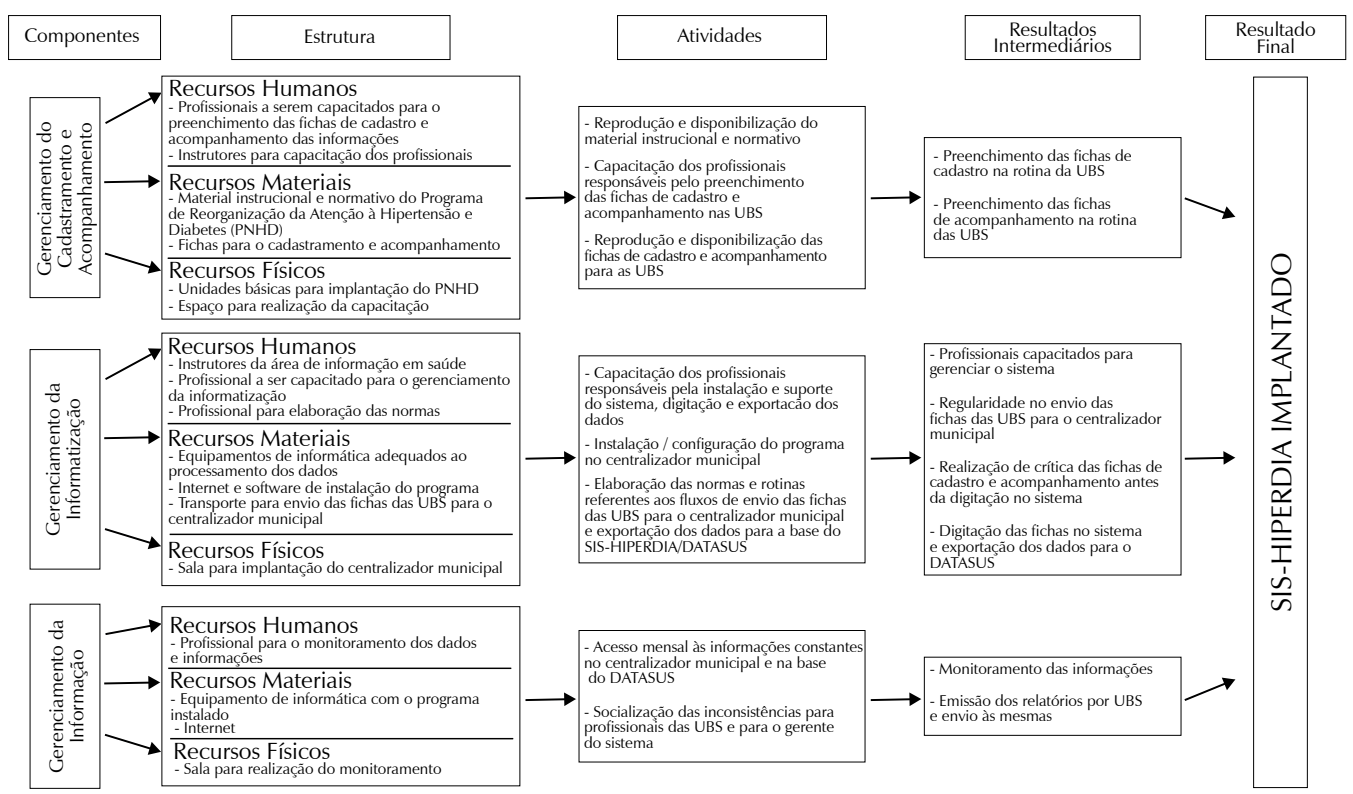

Fonte: Elaboração própria com base no Plano de Reorganização e Atenção ao Diabetes e Hipertenso da Sesab.

Figura 2 - Modelo para análise do contexto da implantação do SIS-HiperDia

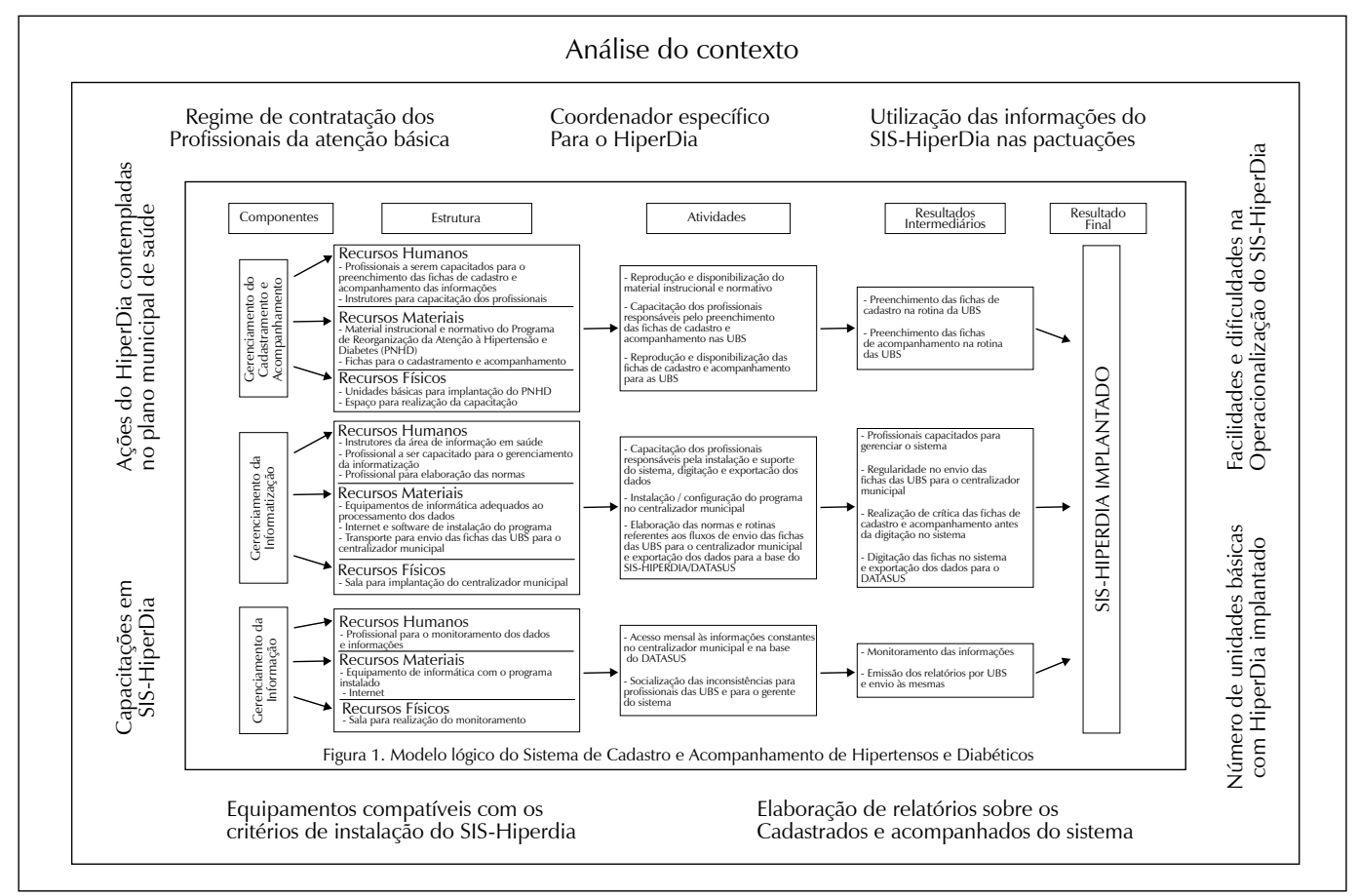

Fonte: Elaboração própria. 
Revista Baiana de Saúde Pública
O segundo passo foi a definição da amostra, formada por conveniência: 143 (34,3\%) municípios, correspondentes à população de 4.476.100 habitantes (31,6\% do total do estado), pertencentes às áreas de abrangência de nove Diretorias Regionais de Saúde (Dires), escolhidas por apresentarem estrutura de organização que facilitou o acesso às secretarias municipais de saúde e, consequentemente, aos respondentes da pesquisa, sendo uma Dires por macrorregião de saúde (Figura 3), de acordo com o Plano Diretor de Regionalização (PDR/ Sesab). Os respondentes foram coordenadores da atenção básica dos municípios.

Figura 3 - Distribuição espacial dos municípios selecionados (143) para análise de implantação do SIS-Hiperdia, de acordo com a Dires e Macrorregiões de Saúde. Estado da Bahia - 2013

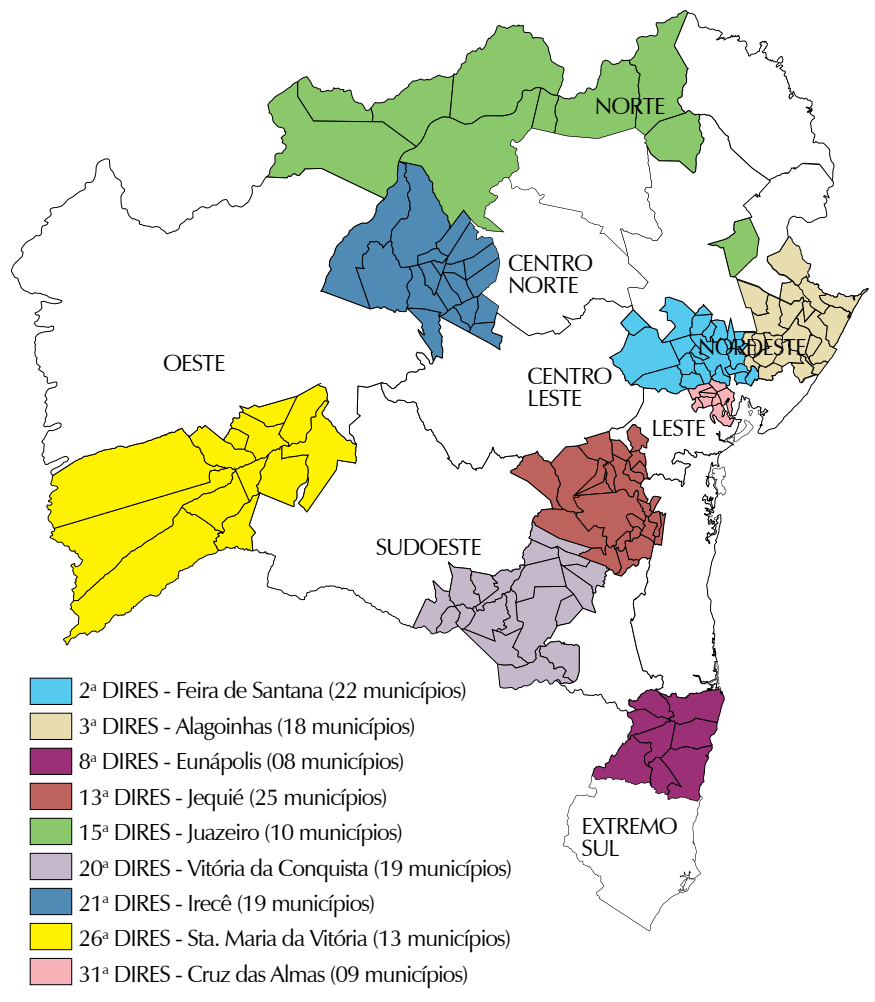

Fonte: Elaboração própria com base no Plano Diretor de Regionalização da Sesab.

A elaboração dos instrumentos para a coleta de dados constituiu o terceiro passo. Construiu-se um questionário para identificar os elementos contextuais e uma planilha do Excel para o cálculo do grau de implantação, a qual contemplava questões referentes a cada componente do SIS-HiperDia. 
O quarto passo consistiu na aplicação de um sistema de escores que possibilitou definir-se o grau de implantação. Esse sistema adotou pesos diferenciados para cada componente, considerando os resultados intermediários de cada um dos componentes. Assim, o gerenciamento do cadastramento e do acompanhamento recebeu peso 2 e a pontuação resultou da soma dos pontos de duas questões. O gerenciamento da informatização também recebeu peso 2 e a pontuação resultou da soma dos pontos de quatro questões. O gerenciamento da informação, por fim, recebeu peso 1 e a pontuação resultou da soma dos pontos de duas questões.

O quinto e último passo foi a análise dos elementos contextuais, que permitiu identificar e discutir as possíveis razões para o grau de implantação encontrado. Foram considerados: regime de contratação dos profissionais da atenção básica; a existência de coordenador específico para o HiperDia; utilização dos dados do SIS-HiperDia nas pactuações; inserção das ações do Programa HiperDia no plano municipal de saúde; realização de capacitações específicas para a operacionalização do SIS-HiperDia; disponibilidade de equipamentos de informática compatíveis com o SIS-HiperDia; periodicidade com a qual os relatórios são gerados; número de unidades básicas do município com HiperDia implantado; e facilidades e dificuldades relatadas pelos respondentes.

Este estudo atendeu a todos os requisitos éticos previstos na Resolução n. 466/2012, do Conselho Nacional de Saúde, e foi aprovado pelo Comitê de Ética em Pesquisa do Instituto de Saúde Coletiva (ISC) da Universidade Federal da Bahia (UFBA), sob Parecer n. 780.348. Os coordenadores municipais que responderam aos questionários assinaram o Termo de Consentimento Livre e Esclarecido (TCLE).

\section{RESULTADOS}

Dos 143 municípios selecionados, 117 (81,8\%) aceitaram participar da pesquisa, $24(16,8 \%)$ não se posicionaram a respeito e $2(1,4 \%)$ referiram não ter condições de responder, por não disporem das informações solicitadas.

De acordo com as respostas obtidas, o SIS-HiperDia estava implantado em 51 (43,6\%) municípios, parcialmente implantado em 60 (51,3\%) e não implantado em 6 (5,1\%).

Com relação ao recorte populacional, 69 municípios (59,0\%) têm menos de 20 mil habitantes; em 25 (36,2\%) deles, o sistema estava implantado; em 38 (55,1\%) parcialmente implantado; e não implantado em 6 (8,7\%). Em 35 (29,9\%) municípios, a população varia entre 20 mil e 50 mil habitantes. Dentre esses $17(48,6)$ tinham o SIS-HiperDia implantado e $18(51,4)$ parcialmente implantado; em $8(6,8 \%)$ municípios, a população varia de 50 a 100 mil habitantes. Nestes, o sistema estava implantado em metade $(4 ; 50,0 \%)$ e parcialmente 
Revista Baiana de Saúde Pública implantado nos outros 4 (50,0\%). Em todos os 5 (4,3\%) municípios com população superior a 100 mil habitantes, o sistema estava implantado.

Os resultados demonstraram que 99\% dos municípios alcançaram a pontuação máxima do componente 1 (gerenciamento do cadastro/acompanhamento), a significar que as fichas eram preenchidas na rotina das Unidades Básicas de Saúde (UBS). Quanto ao gerenciamento da informatização (componente 2), apenas 8,5\% dos municípios alcançaram a pontuação máxima, o que permite concluir-se que as questões referentes à capacitação de profissionais, à crítica e à regularidade do envio das fichas, bem como à exportação dos dados para a base nacional do SIS-HiperDia não foram contempladas ou o foram apenas em parte pela maioria dos municípios.

No que se refere ao componente 3 (gerenciamento da informação), 33\% dos municípios realizavam o monitoramento por meio do acesso ao banco de dados do SIS-HiperDia e geravam os relatórios sobre o perfil dos usuários. Dessa forma, obtiveram os pontos totais definidos para esse componente.

Quanto aos elementos contextuais, discriminam-se os achados a seguir.

Com referência ao regime de contratação, 43 (36,8\%) municípios informaram fazer a contratação temporária dos profissionais da atenção básica; em 9 desses (21,0\%), o SIS-HiperDia estava implantado; em 30 (70,0\%), parcialmente implantado; e em 4 (9,0\%), não implantado. Em 52 (44,4\%) municípios, os profissionais tinham contratação temporária ou eram servidores públicos efetivos; em 32 (61,5\%) desses o sistema estava implantado e em 20 (38,5\%), parcialmente implantado. Foram 10 (8,5\%) os municípios que informaram ter em seus quadros profissionais terceirizados ou efetivos; em 5 (50,0\%) desses, o sistema estava implantado; em 4 (40\%), parcialmente implantado; e em 1 $(10,0 \%)$ não implantado. Apenas 2 (1,7\%) municípios registraram os três tipos de regime de contratação. Em ambos o sistema estava parcialmente implantado. Três (2,6\%) municípios referiram contar com profissionais terceirizados; em 1 (33,3\%) o sistema estava implantado, em 1 (33,3\%) parcialmente implantado e em 1 (33,3\%) não implantado. Foram $4(3,4)$ os municípios que informaram ter profissionais efetivos. Desses, 3 (75,0\%) tinham o SIS-HiperDia implantado e 1 (25,0\%) parcialmente implantado. A contratação temporária ou terceirizada foi informada por 3 (2,6\%) municípios, dos quais 1 (33,3\%) apresentava o sistema implantado e $2(66,7 \%)$ parcialmente implantado (Tabela 1). Os resultados aqui apresentados sugerem que o regime de contratação dos profissionais pode ter influenciado o grau de implantação nos municípios estudados. Naqueles com sistema parcialmente implantado ou não implantado foram predominantes os vínculos considerados precários 
(contratação temporária e terceirizados); nos municípios com sistema implantado, esses vínculos foram proporcionalmente menores.

Tabela 1 - Número de municípios participantes da pesquisa de acordo com o regime de contratação dos profissionais da atenção básica e o grau de implantação alcançado. Estado da Bahia - 2013

\begin{tabular}{|c|c|c|c|c|c|c|c|c|}
\hline \multirow[b]{2}{*}{ Escore } & \multicolumn{7}{|c|}{ Regime de Contratação dos profissionais da atenção básica } & \multirow[b]{2}{*}{ Total } \\
\hline & $\begin{array}{l}\text { Contrata- } \\
\text { ção tem- } \\
\text { porária }\end{array}$ & $\begin{array}{c}\text { Servidor } \\
\text { público } \\
\text { efetivo }+ \\
\text { Contratação } \\
\text { temporária }\end{array}$ & $\begin{array}{l}\text { Servidor pú- } \\
\text { blico efetivo } \\
+ \text { terceiriza- } \\
\text { dos }\end{array}$ & $\begin{array}{l}\text { Servidor } \\
\text { público } \\
\text { efetivo }\end{array}$ & $\begin{array}{l}\text { Contrata- } \\
\text { ção tem- } \\
\text { porária + } \\
\text { terceiriza- } \\
\quad \text { dos }\end{array}$ & $\begin{array}{c}\text { Terceiriza- } \\
\text { dos }\end{array}$ & \begin{tabular}{|c|}
$\begin{array}{c}\text { Servidor } \\
\text { público }\end{array}$ \\
efetivo + \\
Contratação \\
temporária \\
+ Terceiri- \\
zados
\end{tabular} & \\
\hline Implantado & 9 & 32 & 5 & 3 & 1 & 1 & 0 & 51 \\
\hline $\begin{array}{l}\text { Parcialmen- } \\
\text { te implan- } \\
\text { tado }\end{array}$ & 30 & 20 & 4 & 1 & 2 & 1 & 2 & 60 \\
\hline $\begin{array}{l}\text { Não implan- } \\
\text { tado }\end{array}$ & 4 & 0 & 1 & 0 & 0 & 1 & 0 & 6 \\
\hline Total & 43 & 52 & 10 & 4 & 3 & 3 & 2 & 117 \\
\hline
\end{tabular}

Fonte: Elaboração própria.

Quanto ao coordenador do HiperDia, 100 (85,5\%) municípios informaram que não designaram técnico específico e as ações/atividades relacionadas ao programa eram realizadas pelo coordenador da atenção básica. Desses, 43 (43\%) estavam com o sistema implantado, 51 $(51,0 \%)$ parcialmente implantado e 6 (6,0\%) não implantado. Dos 17 (14,5\%) que responderam ter coordenador específico para o programa, $8(47,1 \%)$ estavam com o sistema implantado e $9(52,9 \%)$ parcialmente implantado. A diferença, no que se refere à proporção de municípios com o SIS-HiperDia implantado ou parcialmente implantado, entre o grupo de municípios que tem um coordenador específico e o grupo dos que não o tem não permite conclusão clara sobre a questão: A existência de um coordenador específico é ou não condição favorável para se ter o sistema implantado total ou parcialmente?

A maioria dos municípios, 104 (88,9\%), referiu ter utilizado os dados do SIS-HiperDia nas pactuações. Nesses, o sistema estava implantado em 46 (44,2\%), parcialmente implantado em 54 (52,0\%) e não implantado em 4 (3,8\%). Os outros $13(11,1 \%)$ municípios referiram não ter utilizado as informações do SIS-HiperDia nas pactuações. Desses, 5 (38,5\%) estavam com o sistema implantado, 6 (46,1\%) parcialmente implantado e $2(15,4 \%)$ não 
Revista Baiana de Saúde Pública implantado. Não se observou diferença entre a proporção de municípios que referiram a utilização das informações do SIS-HiperDia nas pactuações e o grau de implantação alcançado. Assim, pode-se considerar que esse elemento não foi influente no contexto da implantação.

No que se refere à inserção das ações do Programa HiperDia no plano municipal de saúde, 52 (44,4\%) municípios responderam ter contemplado essas ações integralmente. Desses, 29 (55,8\%) tinham o sistema implantado, 19 (36,5\%) parcialmente implantado e 4 (7,7\%) não implantado. Dos 64 (54,7\%) que responderam terem incluído parte das ações no plano, 21 (32,8\%) estavam com o sistema implantado, 41 (64,1\%) parcialmente implantado e $2(3,1 \%)$ não implantado. Um município (0,85\%) no qual o sistema estava implantado referiu não ter incluído as ações no plano municipal. Para esse elemento, os resultados indicam que a inclusão das ações do programa HiperDia no plano municipal de saúde não foi influente no processo de implantação do SIS-HiperDia, por não se evidenciarem diferenças entre os graus de implantação alcançados e a proporção de municípios com inclusão total ou parcial das referidas ações nos respectivos planos de saúde.

Apenas 25 (21,4\%) municípios realizaram capacitações específicas para a operacionalização do SIS-HiperDia. Em 17 (68,0\%) deles, o sistema estava implantado, em 5 (20,0\%) parcialmente implantado e em 3 (12,0\%) não implantado. Com relação ao público das capacitações, 13 (52,0\%) municípios informaram terem sido os profissionais da atenção básica, $8(32,0 \%)$ os digitadores e $4(16,0 \%)$ digitadores e profissionais das equipes da atenção básica. Dos 92 (78,6\%) municípios que não realizaram capacitações, em 34 (36,9\%) o sistema estava implantado, em 55 (59,8\%) parcialmente implantado e em 3 (3,3\%) não implantado (Tabela 2). Os resultados sugerem que as capacitações não têm influência na implantação do SIS-HiperDia, total ou parcialmente, mas que são importantes para que a implantação seja completa (total).

Tabela 2 - Número de municípios participantes da pesquisa, de acordo com a realização de capacitações sobre o SIS-HiperDia e o grau de implantação alcançado. Estado da Bahia - 2013

\begin{tabular}{|c|c|c|c|}
\hline \multirow[t]{2}{*}{ Escore } & \multicolumn{2}{|c|}{$\begin{array}{c}\text { Capacitações sobre o } \\
\text { SIS-HiperDia }\end{array}$} & \multirow{2}{*}{ Total } \\
\hline & Sim & Não & \\
\hline Implantado & 17 & 34 & 51 \\
\hline Parcialmente Implantado & 5 & 55 & 60 \\
\hline Total & 25 & 92 & 117 \\
\hline
\end{tabular}


Oitenta e seis municípios (73,5\%) responderam dispor de equipamentos de informática compatíveis com o SIS-HiperDia. Nesses, o grau de implantação foi 43 $(50,0 \%), 39(45,3 \%)$ e $4(4,7 \%)$ para o sistema implantado, parcialmente implantado e não implantado, respectivamente. Dos $27(23,1 \%)$ municípios que informaram dispor em parte de equipamentos compatíveis com o sistema, em 7 (25,9\%) o sistema estava implantado e em $20(74,1 \%)$ parcialmente implantado. Os outros 4 (3,4\%) municípios informaram não dispor de equipamentos compatíveis com os critérios para instalação do SIS-HiperDia; desses, em 1 (25,0\%) o sistema estava implantado, em 1 (25,0\%) parcialmente implantado e em 2 (50,0\%) não implantado. Os achados são contraditórios, por indicarem que o SIS-HiperDia pode ser implantado mesmo sem equipamento algum, o que não seria possível, e que dispor em parte dificulta a implantação total.

A periodicidade com a qual os relatórios eram gerados variou de semanal a anual. A maioria dos municípios $(91 ; 84,3 \%$ ) elaborava-os mensalmente, enviando-os às equipes das UBS ou para o técnico responsável pela operacionalização do sistema como feedback. Desses municípios, 40 (44,0\%) estavam com o sistema implantado, 49 (53,8\%) parcialmente implantado e 2 (2,2\%) não implantado. Nove municípios (7,7\%) não elaboravam relatórios e o número de municípios, 3 (33,3\%), foi o mesmo para cada um dos 3 graus de implantação. Apenas 1 (0,85\%) município informou gerar relatório anualmente e, neste, o sistema estava parcialmente implantado. Dos $6(5,1 \%)$ municípios que referiram periodicidade semestral, em 2 (33,3\%) o sistema estava implantado, em 3 (50,0\%) parcialmente implantado e em 1 (16,7\%) não implantado. A periodicidade foi semanal em 2 (1,7\%) municípios, nos quais o sistema estava implantado em 1 (50\%) e parcialmente implantado em 1 (50\%). A periodicidade foi trimestral em 4 (3,4\%) municípios, nos quais o sistema estava implantado em $3(75,0 \%)$ e parcialmente implantado em 1 (25,0\%). Em 4 (3,4\%) municípios a periodicidade foi quinzenal. Nestes o sistema estava implantado em 2 (50\%) e parcialmente implantado em 2 (50,0\%). Os resultados sugerem que a periodicidade com a qual os relatórios foram gerados não teve influência na implantação (total ou parcial), mas a não elaboração dos relatórios pode ter influenciado a não implantação do sistema.

Para os 117 (100\%) municípios, o Programa HiperDia foi implantado em $100 \%$ das UBS; desses, o SIS-HiperDia estava implantado em 51 (43,6\%), parcialmente implantado em 60 (51,3\%) e não implantado em 6 (5,1\%). A cobertura do programa HiperDia nos municípios estudados não se evidenciou como influente na implantação do sistema, uma vez que a proporção foi a mesma para os implantados total ou parcialmente e não implantados. 
Revista Baiana de Saúde Pública
As facilidades e as dificuldades relatadas pelos coordenadores da atenção básica encontram-se descritas no Quadro 1. Facilidades e dificuldades não foram citadas por 28 (23,9\%) e 15 (12,8\%) municípios respectivamente.

Quadro 1 - Resumo das facilidades e dificuldades relatadas pelos coordenadores da Atenção Básica dos municípios participantes da pesquisa. Bahia, Brasil - 2013

\begin{tabular}{|c|c|c|c|}
\hline Facilidades & $\begin{array}{l}\text { Número de } \\
\text { citações }\end{array}$ & Dificuldades & $\begin{array}{l}\text { Número de } \\
\text { citações }\end{array}$ \\
\hline Digitação e operacionalização do sistema & 37 & $\begin{array}{l}\text { Conectividade, internet e exportação } \\
\text { dos dados }\end{array}$ & 34 \\
\hline Emissão de relatórios & 31 & Preenchimento das fichas & 26 \\
\hline $\begin{array}{l}\text { Acompanhamento/ monitoramento dos } \\
\text { pacientes através do sistema }\end{array}$ & 15 & $\begin{array}{l}\text { Não realização de capacitação para } \\
\text { implantação do programa }\end{array}$ & 18 \\
\hline Preenchimento de fichas & 10 & Reprodução das fichas & 15 \\
\hline Ficha clara/autoexplicativa & 9 & $\begin{array}{l}\text { O sistema foi descontinuado para } \\
\text { implantação do e-SUS-AB }\end{array}$ & 14 \\
\hline Facilidade de alimentação do sistema & 3 & $\begin{array}{l}\text { Impossibilidade de realizar transferência } \\
\text { de usuários entre as UBS e exclusão dos } \\
\text { óbitos }\end{array}$ & 12 \\
\hline Equipamento de informática & 1 & $\begin{array}{l}\text { O sistema não faz interface com outros } \\
\text { sistemas da atenção básica }\end{array}$ & 8 \\
\hline Recursos humanos & 1 & $\begin{array}{l}\text { Falta de suporte para atualização das } \\
\text { versões }\end{array}$ & 7 \\
\hline Profissionais com boa vontade & 1 & $\begin{array}{l}\text { Rotatividade de profissionais nas } \\
\text { equipes, exigindo treinamento para o } \\
\text { preenchimento das fichas. }\end{array}$ & 5 \\
\hline $\begin{array}{l}\text { Condições de trabalho adequadas, com } \\
\text { equipamento, impressos e conectividade } \\
\text { para alimentação do sistema }\end{array}$ & 1 & $\begin{array}{l}\text { Ficha extensa, muitas fichas com as } \\
\text { mesmas informações, muito papel para } \\
\text { preencher }\end{array}$ & 5 \\
\hline $\begin{array}{l}\text { Possibilidade de realizar busca ativa } \\
\text { quando comparado com o SIAB }\end{array}$ & 1 & $\begin{array}{l}\text { Digitação centralizada na Secretaria } \\
\text { Municipal de Saúde }\end{array}$ & 4 \\
\hline $\begin{array}{l}\text { Sistema instalado in-locu facilitando a } \\
\text { alimentação }\end{array}$ & 1 & $\begin{array}{l}\text { Déficit de recursos humanos para a } \\
\text { digitação }\end{array}$ & 4 \\
\hline $\begin{array}{l}\text { Programa específico para o } \\
\text { acompanhamento de hipertensos e } \\
\text { diabéticos }\end{array}$ & 1 & Quantidade de informações & 1 \\
\hline $\begin{array}{l}\text { Apoio por parte dos técnicos da SESAB no } \\
\text { esclarecimento de dúvidas sobre o sistema }\end{array}$ & 1 & $\begin{array}{l}\text { Site do Data SUS fora do ar por muitos } \\
\text { dias, prejudicando a exportação dos } \\
\text { dados }\end{array}$ & 1 \\
\hline
\end{tabular}

Fonte: Elaboração própria. 
A facilidade mais citada por 37 (31,6\%) municípios foi digitação/operacionalização do sistema (campos das fichas de fácil compreensão e compatíveis com a versão on-line/acesso às informações do passo a passo para exportação dos dados no sítio da internet). Desses, 28 $(75,7 \%)$ tinham o sistema implantado e $9(24,4 \%)$ parcialmente implantado. A emissão de relatórios (link disponível na base do sistema que fornecia relatórios com seleção de informações variadas: número de pacientes por patologia, medicamentos em uso, complicações instaladas) foi uma facilidade citada por 35 municípios (29,9\%), dos quais 17 (48,6\%) apresentavam o sistema implantado, $17(48,6 \%)$ parcialmente implantado e 1 (2,8\%) não implantado. $\mathrm{O}$ acompanhamento/monitoramento dos pacientes (acesso às informações sobre os usuários no centralizador municipal por UBS, identificação nominal dos usuários, relação dos óbitos) foi citado como facilidade por 31 municípios (26,5\%). Desses, 4 (12,9\%) tinham o sistema implantado, 26 (83,9\%) parcialmente implantado e 1 (3,2\%) não implantado.

A dificuldade mais relatada por $34(29,1 \%)$ municípios foi a fragilidade na conectividade/internet e na exportação de dados, dos quais $24(70,6)$ tinham o sistema implantado, 9 (26,5\%) parcialmente implantado e $1(2,9 \%)$ não implantado. O não preenchimento das fichas foi citado por 26 municípios (22,2\%); desses, 14 (53,8\%) tinham o sistema implantado e $12(46,2 \%)$ parcialmente implantado. A não realização de capacitação para implantação do programa foi referida por $18(15,4)$ municípios, dos quais $6(33,3 \%)$ tinham o sistema implantado, 9 (50,0\%) parcialmente implantado e $3(16,7 \%)$ não implantado. Registre-se, por um lado, que a digitação/operacionalização pode ter influenciado favoravelmente a implantação total do sistema, o acompanhamento/monitoramento dos pacientes, a implantação parcial e a emissão de relatórios tanto a implantação parcial quanto a total. Por outro, a não realização de capacitações pode ter dificultado a implantação parcial.

\section{DISCUSSÃO}

Os resultados demonstraram que, nos locais em que o sistema não estava implantado $(66,7 \%)$, foi maior a proporção de municípios nos quais ocorria a precarização dos vínculos empregatícios: em 50\%, o sistema estava parcialmente implantado; e em 17,6\%, não implantado. Isso sugere que o regime de contratação dos profissionais pode ter influenciado o grau de implantação. Em estudo recente14, os autores afirmam que as mudanças nas gestões municipais acarretam a rotatividade dos profissionais que atuam no programa HiperDia.

Quanto à definição de coordenador específico para o HiperDia, cem municípios não o possuem. Destes 43\% estão com o sistema implantado; $51 \%$ parcialmente implantado e $6 \%$ não implantado (corresponde a 100\% desse grau de implantação). 
Revista Baiana de Saúde Pública
Os achados não esclarecem se o fato de se definir ou não um coordenador específico para o programa HiperDia foi elemento influente para a implantação total ou parcial, mas indicam que não se ter o coordenador específico pode ser a razão de municípios não o terem implantado. Outros estudos ${ }^{14-15}$ fazem referência à inexistência de coordenador do HiperDia em muitos municípios e que os dados são gerados sem o devido treinamento ou supervisão.

Quanto ao uso das informações do SIS-HiperDia nas pactuações, não foram observadas associações entre o uso ou não das informações e o grau de implantação alcançado. Assim, esse elemento contextual não foi influente. É importante ressaltar que, com o objetivo de avaliar de forma indireta as ações básicas de prevenção e controle da HAS e DM, a proporção de hipertensos e diabéticos cadastrados no SIS-HiperDia passou a integrar o elenco de indicadores da Atenção Básica em 2006.

Resultado semelhante foi encontrado quanto à inclusão das ações do HiperDia no plano municipal de saúde. Entretanto, não foram observadas associações entre a inclusão ou não das ações do HiperDia no plano municipal de saúde e o grau de implantação alcançado. Registre-se que esse plano constitui elemento importante, no contexto da implantação do sistema, para se assegurar os recursos (humanos, físicos e materiais) necessários à operacionalização do SIS-HiperDia.

Apenas $25(21,4 \%)$ municípios realizaram capacitações específicas para a operacionalização do SIS-HiperDia. Não ter realizado capacitações reduziu a pontuação do componente 2 no cálculo do escore em 0,5 ponto, do total de 4. Estudos ${ }^{14,16-17}$ ressaltam a importância de treinamento para o uso do SIS-HiperDia.

Quanto à disponibilidade de equipamentos de informática compatíveis com o SIS-HiperDia, os achados são contraditórios, por indicarem que esse sistema pode ser implantado mesmo sem equipamento algum, o que não seria possível, e que dispor em parte dificulta a implantação total. Dois dos municípios (um com implantação total do sistema e outro com implantação parcial) informaram não ter os equipamentos. A falta dos equipamentos básicos de informática foi citada como uma das principais dificuldades para implantação e adequado funcionamento do sistema ${ }^{14,18}$.

Cento e oito municípios elaboravam relatórios, 84,3\% tinham periodicidade mensal. Não ter elaborado relatórios pode ter sido um elemento influente nos municípios com sistema não implantado. Assim, os resultados sugerem que a elaboração dos relatórios foi importante, mas que a sua periodicidade não influenciou o grau de implantação neste estudo. 
A cobertura do programa HiperDia nos municípios estudados não se evidenciou como influente na implantação do sistema, uma vez que a proporção foi a mesma para os implantados total ou parcialmente e não implantados, embora se pudesse supor que uma maior cobertura do programa poderia influenciar positivamente o grau de implantação, traduzindo maior apoio da gestão e condução das ações em cada município.

O registro de facilidades e dificuldades pelos respondentes revelou outros aspectos do contexto relacionados à qualidade das fichas, conectividade, atualização das versões, impossibilidade de transferência dos usuários entre UBS e inexistência de interface com outros sistemas, como influentes no grau de implantação, dadas as características da gestão e a condução das ações em cada município. Também reforçou a influência positiva da emissão de relatórios, bem como da falta de capacitação e dos aspectos informacionais como influência negativa para o grau de implantação do SIS-HiperDia.

\section{CONSIDERAÇÕES FINAIS}

O estudo permitiu concluir-se que o grau de implantação do SIS-HiperDia nos municípios baianos analisados foi baixo. Considerando que a HAS e o DM estão entre as principais DCNT e constituem grande problema de saúde pública, esse baixo grau é revelador da fragilidade do sistema de saúde nesses municípios. Com efeito, a implantação de um sistema que possibilita gerar informações sobre o cadastramento e o acompanhamento dos usuários acometidos pelas patologias em questão seria de fundamental importância para a análise da situação de saúde e proposição de estratégias de prevenção e controle.

A implantação do SIS-HiperDia nos municípios estudados apresentou dificuldades informacionais e gerenciais. Este estudo permitiu identificar fragilidades no gerenciamento, as quais podem repercutir diretamente na qualidade da informação e no seu uso como ferramenta de planejamento e de gestão do cuidado em saúde.

A não realização de capacitação dos técnicos, em especial, está a comprometer a fidedignidade das informações e, portanto, o alcance dos objetivos para o qual o sistema foi criado. A não disponibilização de recursos materiais, equipamentos e as falhas na conectividade, que dificultam ou impedem o processo de digitação e exportação dos dados, são obstáculos que podem e devem ser superados, permitindo a melhoria da qualidade da atenção a hipertensos e diabéticos.

Enfim, este estudo convida a refletir sobre o uso da informação gerada pelos sistemas, os quais sofrem a influência de vários aspectos relacionados à sua implantação e manutenção e que balizam decisões para o estabelecimento de políticas públicas no SUS. 
Revista Baiana de Saúde Pública

\section{COLABORADORES:}

1. Concepção do projeto, análise e interpretação dos dados: Edna Pereira Rezende e Luis Eugênio Portela Fernandes de Souza.

2. Redação do artigo e revisão crítica relevante do conteúdo intelectual: Edna Pereira Rezende e Luis Eugênio Portela Fernandes de Souza.

3. Revisão e/ou aprovação final da versão a ser publicada: Edna Pereira Rezende.

4. Ser responsável por todos os aspectos do trabalho na garantia da exatidão e integridade de qualquer parte da obra: Edna Pereira Rezende e Luis Eugênio Portela Fernandes de Souza.

\section{REFERÊNCIAS}

1. Goulart FAA. Doenças crônicas não transmissíveis: estratégias de controle e desafios e para os sistemas de saúde. Brasília: OPAS; 2011.

2. Schmidt MI, Duncan BB, Silva GA, Menezes AM, Monteiro CA, Barreto SM, et al. Doenças crônicas não transmissíveis no Brasil: carga e desafios atuais. Série Saúde no Brasil 4. Lancet. 2011 maio;4:61-74.

3. Organização Mundial de Saúde. Cuidados inovadores para condições crônicas: componentes estruturais de ação. Relatório mundial. Brasília; 2003.

4. Brasil. Ministério da Saúde. Doenças Cônicas não Transmissíveis: Vigilância de doenças crônicas não transmissíveis. Portal saúde. Brasília; 2012 [citado 2014 abr 15]. Disponível em: http://portal.saude.gov.br/portal/saude/profissional/ visualizar_texto.cfm?idtxt $=3187$

5. Passos VMA, Assis TD, Barreto SM. Hipertensão arterial no Brasil: estimativa de prevalência a partir de estudos de base populacional. Epidemiol serv saúde. 2006 jan/mar;15(1):35-45.

6. Brasil. Ministério da Saúde. Secretaria de Vigilância em Saúde. Departamento de Vigilância de Doenças e Agravos não Transmissíveis e Promoção da saúde. Vigitel Brasil 2013: vigilância de fatores de risco e proteção para doenças crônicas por inquérito telefônico. Brasília; 2014. [citado 2014 abr 10]. Disponível em: bvsms.saude.gov.br/bvs/vigitel_ brasil_2013_vigilancia_risco.pdf

7. Brasil. Ministério da Saúde. Prevenção e cuidado integrados para o controle da hipertensão arterial e diabetes mellitus. Nota Técnica n. 26. Brasília; 2011.

8. Bahia. Secretaria da Saúde do Estado da Bahia. Boletim epidemiológico de doenças crônicas não transmissíveis (DCNT) n. 1. Salvador; 2014. [citado 2013 dez 14]. Disponível em: www.suvisa.ba.gov.br/vigilanciaepidemiologica 
9. Brasil. Ministério da Saúde. Coordenação Nacional de HA e DM.

Departamento de Atenção Básica. Secretaria de Atenção a Saúde.

Apresentação do HiperDia. Brasília; 2006. [citado 2013 dez 14]. Disponível em: http://hiperdia.datasus.gov.br/apresentacao.asp

10. Brasil. Ministério da Saúde. Vigitel Brasil 2012. Biblioteca Virtual do Ministério da Saúde. Brasília; 2013. [citado 2013 dez 14]. Disponível em: bvsms.saude. gov.br/bvs/vigitel_brasil_2012_vigilancia_risco.pdf

11. Brasil. Ministério da Saúde. Relatórios gerados pelo Sistema de Informação HiperDia do Ministério da Saúde, como forma de instrumento de Gerenciamento da Atenção à Hipertensão Arterial e ao Diabetes Mellitus para capacitar profissionais de saúde de nível superior da rede básica. Brasília; 2008 jun. BR/CNT/0700644.001.

12. Brasil. Ministério da Saúde. Plano de Reorganização da Atenção à Hipertensão Arterial e ao Diabetes mellitus. Brasília; 2001.

13. Brasil. Ministério da Saúde. Manual de Operação do HiperDia. Sistema de cadastramento de acompanhamento de hipertensos e diabéticos. Versão 1.5 M02. Rio de Janeiro; 2002.

14. Chazan AC, Perez EA. Avaliação da implantação do sistema informatizado de cadastramento e acompanhamento de hipertensos e diabéticos (HIPERDIA) nos municípios do estado do Rio de Janeiro. Rev APS. 2008 janmar;11(1)10-6.

15. Silva AS, Laprega MR. Avaliação crítica do Sistema de Informação da Atenção Básica (SIAB) e de sua implantação na região de Ribeirão Preto, São Paulo, Brasil. Cad Saúde Pública; 2005 nov-dez;21(6):1821-8.

16. Oliveira CA, Palha PF. Sistema de informações HiperDia, 2002-2004, adequações das informações. Cogitare Enferm. 2008 jul/set;23(3):395-402.

17. Jardim ADI, Leal AMO. Qualidade da informação sobre diabéticos e hipertensos registrada no Sistema HIPERDIA em São Carlos-SP, 2002-2005. Rev Saúde Coletiva. 2009;19(2):405-17.

18. Sena VL. Processo de alimentação do Sistema de Informação do HiperDia: estudo de caso no município de Natal/RN (dissertação). Natal: Universidade Federal do Rio Grande do Norte; 2008 [citado 2014 maio 10]. Disponível em: https://repositorio.ufrn.br/jspui/bitstream/123456789/14723/1/VivianeLS_ DISSERT.pdf

Recebido: 25.7.2015. Aprovado 8.8.2015. Publicado: 20.9.2017. 